\title{
The effect on forearm and shoulder muscle activity in using different slanted computer mice
}

\author{
Han-Ming Chen *, Chun-Tong Leung \\ Department of Mechanical Engineering, National Taiwan University, Taipei 106, Taiwan, ROC
}

Received 6 September 2006; accepted 3 January 2007

\begin{abstract}
Background. Frequently using non-slanted computer mice will cause musculoskeletal discomfort and symptoms in forearms and shoulders. Ergonomic mice with different slanted angles may have various effects on forearm and shoulder muscle activity.

Methods. All of the twelve subjects performed the same text-editing task with the five different slanted mice. The muscle activity of extensor carpi ulnaris, extensor digitorum, pronator teres and upper trapezius muscles was recorded by surface electromyography and analyzed by a non-parametric method.

Findings. As the slanted angles increased, the surface electromyography levels in terms of extensor carpi ulnaris, pronator teres and upper trapezius muscles decreased. However, increasing the slanted angles resulted in larger wrist extension and higher muscle activity in terms of the extensor digitorum muscle.

Interpretation. Working with mice which have suitable slanted angles provides users more neutral hand positions, so forearm and shoulder muscle activity and the risk of musculoskeletal disorders will reduce.

(C) 2007 Elsevier Ltd. All rights reserved.
\end{abstract}

Keywords: Ergonomic mouse; Slanted surface; Forearm and shoulder muscle activity; Surface electromyography

\section{Introduction}

Over two-thirds of all occupational disorders recognized in France were work-related musculoskeletal disorders of the upper limb, which were mainly induced by biomechanical factors such as repetitive motion, strenuous effort, and extreme joint postures (Aptel et al., 2002). The risk of musculoskeletal disorders was greater among workers who used computers in uncomfortable positions for long time (Ortiz-Hernández et al., 2003). According to the study proposed by Blatter and Bongers (2002), people whose working time with computers was more than $6 \mathrm{~h}$ per day were strongly associated with upper limb disorders in some body regions, e.g. neck, shoulder, elbow, arm or wrist/hand. The elbow, arm or wrist/hand disorders of computer users who

\footnotetext{
${ }^{*}$ Corresponding author.

E-mail address: hmchen@ntu.edu.tw (H.-M. Chen).
}

worked with mice for $6-8 \mathrm{~h}$ per day were moderately greater than that of computer users who worked without mice. As the computer mouse became more and more indispensable to modern software, intensive mouse users might suffer from musculoskeletal symptoms in the neck and upper extremity (Cook et al., 2000). In the research reported by Atkinson et al. (2004), nearly half of the mouse users who used the mouse $6 \mathrm{~h}$ per day on average reflected musculoskeletal pain and discomfort especially in wrists, hands and fingers. Jensen et al. (2002) also observed that employees working with intensive mouse use experienced a stronger association with hand/wrist symptoms than those working with computers but without mice.

From the above studies, musculoskeletal symptoms might occur while using the mouse in non-neutral upper limb postures. People who used computer mice and trackballs were exposed to extreme ulnar deviation and wrist extension (Burgess-Limerick et al., 1999). Mouse operators showed more strenuous working postures, especially in the 
wrist, elbow and shoulder, than non-mouse operators (Karlqvist et al., 1994). A non-slanted mouse is always used under considerable forearm pronation and wrist deviation that are considered to be potential risk factors for musculoskeletal illness in the elbow and forearm. Pronation from the vertical plane over $60^{\circ}$ would result in a steep increase in forearm muscle activity (Zipp et al., 1983). The neutral zone of the wrist was $5-7^{\circ}$ ulnar deviation and $7-9^{\circ}$ extension, and the muscle activity in this neutral zone could reduce up to $75 \%$ of that in specified wrist deviated postures (Fagarasanu et al., 2004). Referring to the study carried by Karlqvist et al. (1994), mouse operators who worked with the wrist deviating in the range from $-5^{\circ}$ to $60^{\circ}$ had $64 \%$ of the working time with the wrist deviating more than $15^{\circ}$ towards the ulnar side. By contrast, keyboard operators had $96 \%$ of the working time with the wrist in the neutral zone slightly towards radial deviation. Due to different forearm working postures, the probability of having upper-limb disorders in workers doing mouseintensive tasks was higher than those doing keyboardintensive tasks.

Fagarasanu and Kumar (2003) found that carpal tunnel syndrome (CTS) was a work related to upper extremity disorder and could cause serious impact to the health of computer users. CTS pathogenesis was due to elevated carpal tunnel pressure (CTP) which was caused by prolonged awkward postures such as wrist flexion/extension, forearm pronation/supination or ulnar/radial deviation. Werner et al. (1997) also observed that the intra-carpal canal pressure (ICCP) increased with wrist flexion/extension, forearm pronation/supination and ulnar/radial deviation. Elevated ICCP could be arisen from deformed tissues in the wrist. Wrist extension would stretch the tissues in the carpal tunnel, whereas forearm pronation would shear the tissues in the wrist and wind them tightly around the tunnel. It would cause CTS with the median nerve in the tunnel being stretched, squeezed or twisted when the wrist was in prolonged extreme postures (Buckle, 1997). In comparison between mouse use and keyboard use among participants in the one-year follow-up study, it was found that the risk of possible CTS was associated with mouse use for more than $20 \mathrm{~h}$ per week, but that was not statistically significantly associated with keyboard use (Anderson et al., 2003).

Several studies had devoted to compare forearm and shoulder muscle activity in using non-slanted mice with that in using ergonomic mice (Aarås and Ro, 1997; Agarabi et al., 2004; Gustafsson and Hagberg, 2003). The results of the above three studies revealed that lower muscle activity was observed in carpi ulnaris (ECU), extensor digitorum (ED), pronator teres (PT) and upper trapezius (Trap) muscles, etc. while using the ergonomic mice. The pains in the neck, shoulder, forearm and wrist/hand, which were caused by using the non-slanted mouse, were gradually reduced after a considerable period of time in using the ergonomic mouse (Aarås et al., 2002). The aim of this study was to try to examine the effect on the activity of four muscles while using five different slanted mice with surface electromyography (sEMG).

\section{Methods}

\subsection{Subjects}

After informed consent, 9 males (age 23.8 [2.82] (20-30) years, height 174.6 [6.86] $(166-183) \mathrm{cm}$ and weight 69.8 [11.6] (53-95) kg) and 3 females (age 24.0 [1.00] (23-25) years, height 163.3 [4.16] $(160-168) \mathrm{cm}$ and weight 52.3 [2.52] $(50-55) \mathrm{kg}$ ) participated voluntarily in this experiment. The mean values and standard deviations of the subject data are listed before and in the brackets, respectively. All subjects were right-handed users, free of wrist/forearm and shoulder pain, and familiar with computer mouse usage.

\subsection{Tasks and measurements}

\subsubsection{Apparatus}

According to the investigation carried out by Johnson et al. (1996), the surface EMG was suitable and feasible to assess forearm and shoulder muscle activity. EMG raw data were recorded by Motion Lab Systems (LA, USA) MA300 system with bipolar $\mathrm{Ag} / \mathrm{AgCl}$ surface electrodes (MA-311, Motion Lab Systems, LA, USA) on which two recording areas were $18 \mathrm{~mm}$ apart.

In this experiment, we had fabricated five custom-made mice with slanted angles of $0^{\circ}, 10^{\circ}, 20^{\circ}, 25^{\circ}$ and $30^{\circ}$ and weights of $80.7,80.5,86.5,90.1$ and $100.1 \mathrm{~g}$ (conducting wires excluded), respectively. In Fig. 1, the angle of the slanted surface is defined as the angle between a horizontal plane and the inclined surface of a mouse in the front or rear views. As shown in Fig. 2, these five slanted mice have the same base shape and right-side height.

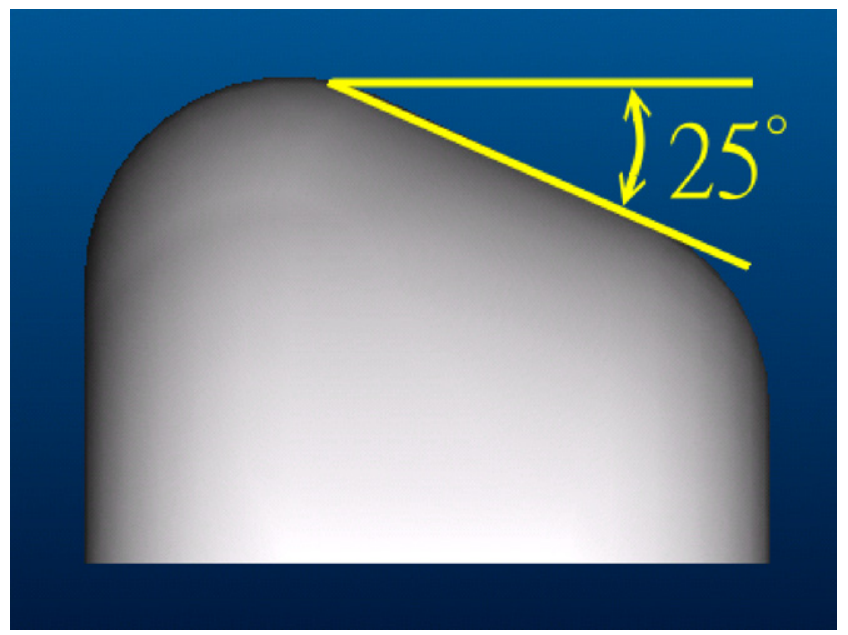

Fig. 1. The rear view of the $25^{\circ}$ slanted mouse model. 


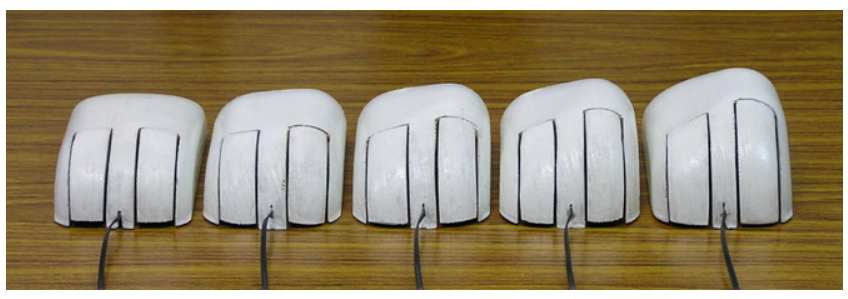

Fig. 2. Five tested mice with slanted angles of $0^{\circ}, 10^{\circ}, 20^{\circ}, 25^{\circ}$ and $30^{\circ}$ from left to right.

\subsubsection{Experimental procedure}

In order to prevent the existing forearm and shoulder muscle fatigue before the experiment, all the subjects were advised to avoid high-intensity sport or any activity that might cause tiredness of the upper extremity two days before testing. The room temperature throughout the whole experiment was kept around $25{ }^{\circ} \mathrm{C}$ to reduce the experimental error from temperature.

The subject sat upright in a height-adjustable office chair, with feet placed flat on the floor, and rested both forearms on the desk. Before the test was processed, the height of the seat should be adjusted to fit the subject. The mouse was operated generally on the right side of the keyboard. With the forearm resting on the tabletop, abduction of the arm was checked and avoided in order to reduce posture load in terms of the Trap muscle (Harvey and Peper, 1997).

Cram et al. (1998) recommended that single differential electrodes should be applied to the muscle belly in the direction parallel to the muscle fibers. The electrode of the ED muscle was applied to the middle of the forearm approximately three quarters of the distance from the elbow to the wrist while the subject extended his fingers. The ECU muscle was palpated for the active muscle mass on the ulnar side of the forearm at a few centimeters below the elbow while the subject was asked to do ulnar deviation. The PT muscle was palpated for the active muscle mass around the soft valley in the middle of the ventral aspect of the forearm just below the elbow while the subject pronated (palm-up to palm-down) the forearm. The electrode of the Trap muscle was placed along the ridge of the shoulder slightly lateral to and one half the distance between the cervical spine at C-7 and the acromion.

Four electrodes were adhered to the skin by adhesive tape with proper tightness. Skins where the EMG electrodes were cohered were rubbed with alcohol cotton balls until redness to reduce electrode-skin impedance (Clancy et al., 2002). The signal of each EMG channel was tested for normal operation and data acquisition.

\subsubsection{Data acquisition}

EMG raw data were sampled at a rate of $1800 \mathrm{~Hz}$. The EMG band-pass filter was set between $20 \mathrm{~Hz}$ and $450 \mathrm{~Hz}$ with a $60 \mathrm{~Hz}$ notch filter.

The experimental task consisted of highlighting and copying the text in each odd-number line and then pasting the text into the next blank line with the mouse in approximately constant speed. Each subject was asked to perform a 30-min task with each of the five tested mice in random order. EMG data were recorded in the last $30 \mathrm{~s}$ for each 30 -min task. Five 30-min tasks were spaced by 10 -min breaks to reduce the cumulative effect of muscle fatigue. During the test, each subject was advised to rest the whole palm on the mouse to obtain an apparent effect on muscle activity while using the different slanted mice. Subjective ratings were not under consideration because ratings seemed useless on judging ergonomic devices in low-intensity tasks (Visser et al., 2000).

\subsubsection{Data analysis}

For each of PT, ECU, ED and Trap muscles, the highest EMG value of the maximum voluntary isometric contraction was denoted as the maximal voluntary electrical activity (MVE) which had good test-retest repeatability (Bao et al., 1995). After processing, the mean value of the EMG root mean square (RMS) data was calculated for each task and then normalized against MVE.

The normalized data were analyzed by SPSS 10.0 (SPSS Inc., Chicago, USA) statistical software. For lack of a normal distribution, the data were analyzed by a nonparametric method. The significant difference between each of the four slanted mice and the non-slanted mouse was analyzed by the Wilcoxon signed-rank test. Statistical significance was set at $P<0.05$.

\section{Results}

Fig. 3 presents all normalized mean EMG values of the 12 subjects in terms of the four examined muscles after having used each of the five slanted computer mice for $30 \mathrm{~min}$. ECU and Trap muscles had the same tendency of decreas-

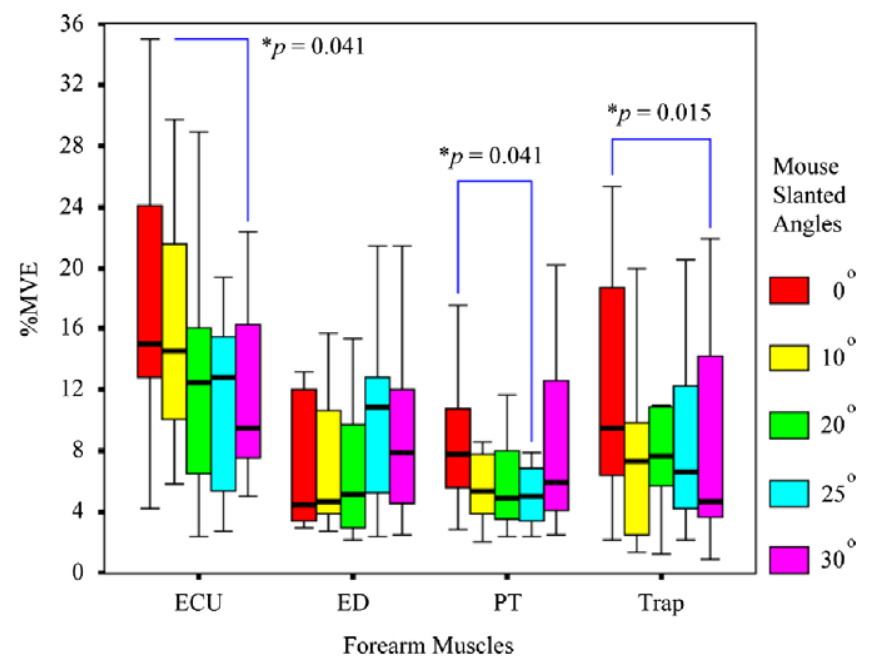

Fig. 3. The normalized mean EMG values of the 12 subjects in terms of the four upper-extremity muscles after five 30-min mouse tasks separately: extensor carpi ulnaris; extensor digitorum; pronator teres; and upper trapezius. An asterisk indicates a significant difference. Boxes were given as mean values with $50 \%$ confidence intervals from 25 th to 75 th percentile. 
Table 1

The significance level of differences between each normalized muscle activity in using each of the four slanted mice and that in using the nonslanted mouse after 30-min mouse tasks

\begin{tabular}{lllll}
\hline Muscles & \multicolumn{4}{l}{$P$-values (each slanted mouse vs. the non-slanted mouse) } \\
\cline { 2 - 5 } & $10^{\circ}$ vs. $0^{\circ}$ & $20^{\circ}$ vs. $0^{\circ}$ & $25^{\circ}$ vs. $0^{\circ}$ & $30^{\circ}$ vs. $0^{\circ}$ \\
\hline ECU & 0.182 & 0.108 & 0.084 & 0.041 \\
ED & 0.433 & 0.239 & 0.136 & 0.480 \\
PT & 0.136 & 0.224 & 0.041 & 0.583 \\
Trap & 0.530 & 0.084 & 0.182 & 0.015 \\
\hline
\end{tabular}

ing muscle activity as the slanted angles increased. For ECU and Trap muscles, the EMG values in using the $30^{\circ}$ slanted mouse (median values 9.51\% MVE and 4.66\% MVE, respectively), were significantly lower $(P=0.041$ and 0.015 , respectively) compared with that in using the non-slanted mouse as shown in Table 1. The EMG raw data of the Trap muscle of one subject for several seconds in the end of 30-min tasks while using the non-slanted
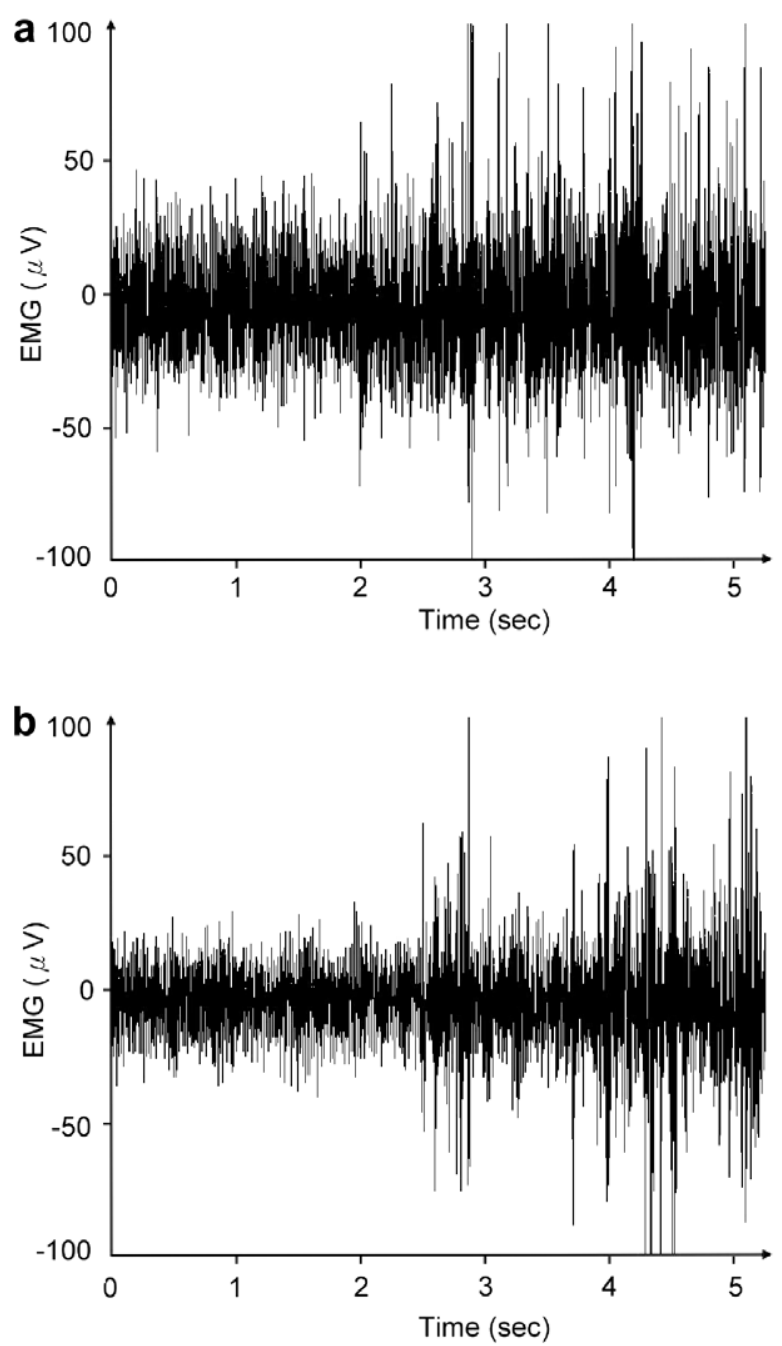

Fig. 4. The EMG raw data of the Trap muscle of one subject for several seconds in the end of 30-min tasks in using (a) the non-slanted mouse $(2 \mathrm{nd} / 5)$ and $(\mathrm{b})$ the $30^{\circ}$ slanted mouse $(3 \mathrm{rd} / 5)$. mouse (the second one of 5 mice) and the $30^{\circ}$ slanted mouse (the third one of 5 mice) are displayed in Fig. 4. The PT muscle revealed a minimum muscle activity (median value $5.04 \% \mathrm{MVE}$ ) in using the $25^{\circ}$ slanted mouse. For the PT muscle, the EMG values in using the $25^{\circ}$ slanted mouse were significantly lower $(P=0.041)$ compared with that in using the non-slanted mouse. Unlike the above three muscles, there was no obvious tendency found in terms of the ED muscle. The EMG values of the ED muscle approximately increased with the slanted angles, and the lowest muscle activity (median value 4.49\% MVE) appeared in using the non-slanted mouse.

\section{Discussion}

Laursen and Jensen (2000) observed that the muscle activity in terms of surface EMG was affected by age. Compared with the young, elder people had higher muscle activity during the computer mouse task. For this reason, the subjects whose ages were within a narrow range were invited to join this study to avoid the experimental error from age.

In fact, there was the cumulative effect of muscle fatigue on the tested muscles no matter how low the workload was and how short the period of the task was. The cumulative effect of muscle fatigue would affect the initial value of muscle activity in each mouse task. For this reason, we tested the five mice in random order to get more objective comparisons. Repetitively working in cold temperature was found to invariably accelerate muscle fatigue and cause higher EMG activity than that in warm temperature (Oksa et al., 2002). To avoid an error from temperature, the room temperature must be controlled around a constant throughout this experiment.

In general, using a mouse primarily consists of wrist extension and finger motion, ulnar/radial deviation, forearm pronation/supination, and shoulder motion which are mainly related to ED, ECU, PT, and Trap muscles, respectively (Baillif and Kimmel, 1945). The results of this study depicted in Fig. 3 reveal that the medians of ECU muscle activity decreased as the slanted angles of the tested mice increased. Because the palm of each subject was asked to rest on the mouse, forearm pronation decreased as the slanted angles increased. Extreme pronation (approximate $90^{\circ}$ pronation) caused pure ulna/radial deviation and neutral hand position (approximate $0^{\circ}$ pronation and $0^{\circ}$ supination) caused pure wrist flexion/extension when the hand moved the mouse laterally and the forearm kept still. Decreasing forearm pronation resulted in decreasing ulna/radial deviation but increasing wrist flexion/extension while dragging the mouse laterally. Hence, dragging a mouse with larger slanted angle led to a decrease in ulna/ radial deviation and ECU muscle activity.

As the slanted angles decreased and the forearm pronation increased, the ventral aspect of the forearm faced downward, so the flexor carpi radialis (FCR) muscle was shortened and bulged, and this caused the elbow to lift 
up from the desk. When the mouse was moved laterally, this bulged part rolling laterally on the desk led to the lateral movement of the elbow. Hence, shoulder abduction/ adduction induced the motion of the Trap muscle. By contrast, as the slanted angles increased, the pronation of the forearm reduced. So the ulnar side gradually faced downward. Because there was no bulged part along the ulnar side, the elbow would touch the desk and become the main part to support the weight of the upper arm. In this situation, the elbow would be the rotation pivot of the forearm when the mouse was moved laterally so the motion of the Trap muscle greatly reduced. The non-slanted and $30^{\circ}$ slanted mice were tested in the order of the second one and the third one of the five tested mice, respectively, for one subject. The EMG amplitude or muscle activity of the Trap muscle of this subject in using the non-slanted mouse was obviously higher than that in using the $30^{\circ}$ slanted mouse as shown in Fig. 4.

In the EMG values in terms of the PT muscle, a lowest median value was found while using the $25^{\circ}$ slanted mouse. As the slanted angles increased, PT muscle activity decreased with forearm pronation in the first four tested mice. It implied that the PT muscle was in a more neutral status when the forearm was pronated by the $25^{\circ}$ slanted mouse than that by the other four mice. In other words, the PT muscle might contract or be stretched away from this neutral status in using any of the other four mice. Zipp et al. (1983) found that PT muscle activity in using the $30^{\circ}$ laterally inclined keyboard was lower than that in using the $10^{\circ}$ or $20^{\circ}$ laterally inclined keyboards. The PT muscle activity in using a mouse or keyboard with $25^{\circ}$ or $30^{\circ}$ slanted surfaces was lower than that with smaller slanted surfaces.

Unlike ECU, Trap and PT muscles, there was no apparent tendency in ED muscle activity. The larger the slanted angle of the tested mouse was, the higher the rough detection of the EMG level of the ED muscle was. The five tested mice were designed with a constant height on the right side, but had different slanted angles. Consequently, increasing the slanted angle caused an increase in the height on the left side of the mouse and in the height of the forefinger and the middle finger. This would increase forefinger, middle finger and wrist extension. Therefore, the wrist and fingers clicking and dragging the mouse were in more muscle contraction, so a higher EMG level of the ED muscle was detected. Furthermore, Werner et al. (1997) found that wrist extension would induce the tissues stretched on the palmar side, so pressure was applied to the underlying tissues of the carpal canal. The greatest ICCP was observed in the motions of wrist flexion/extension, ulna/radial deviation, and forearm pronation/supination. Increasing wrist extension with the slanted angles was out of our anticipation and would be a risk for musculoskeletal symptoms.

From the observation on the EMG values, using the $25^{\circ}$ or $30^{\circ}$ slanted mice was more comfortable than using the other three slanted mice for ECU, Trap and PT muscles.
However, higher EMG values which implied higher wrist extension were found in terms of the ED muscle in using the $25^{\circ}$ or $30^{\circ}$ slanted mice. Hence, we recommend the following two ways to decrease wrist extension when users choose an ergonomic mouse. The height on the right side of the mouse should be as low as possible. For a specific slanted angle of a mouse, a lower height on the right side of the mouse will induce a lower height of the top of the mouse so wrist extension will decrease. An elongated inclined part on the rear side of the mouse will also be very helpful. For a fixed height on the top of a mouse, the wrist can rest on the elongated inclined part and be lifted, so the wrist extension and intra-carpal canal pressure will reduce. If the weights of the five tested mice could be made equal, the $25^{\circ}$ and $30^{\circ}$ slanted mice might have better performances on the four examined muscles than that in this paper.

\section{Conclusions}

In this study, the results showed that the activity of the four examined muscles was affected by the slanted angles of our designed ergonomic mice in repetitive, long duration computer mouse tasks. Among the five tested mice, the $25^{\circ}$ or $30^{\circ}$ slanted mice caused lower muscle activity and more neutral working postures for ECU, Trap and PT muscles. For the ED muscle, a larger slanted angle increased the height of the tested mouse, and this might lead to larger wrist extension and a higher risk of CTS. There are two independent ways to decrease this wrist extension: one way is to choose an ergonomic mouse with a lower right-side height for a specified slanted angle, the other way is to choose a mouse with an elongated rear inclined part on which the wrist can rest and be lifted.

\section{Acknowledgments}

We gratefully acknowledge the support of the National Science Council of the Republic of China to this research under the Grant NSC 92-2212-E-002-078, and specially thank Prof. Tung-Wu Lu for lending us the experimental apparatus, and Prof. Pei-Fang Tang and Prof. Jau-Yih Tsauo for giving us many valuable suggestions.

\section{References}

Aarås, A., Ro, O., 1997. Workload when using a mouse as an input device. International Journal of Human-Computer Interaction 9, 105-118.

Aarås, A., Dainoff, M., Ro, O., Thoresen, M., 2002. Can a more neutral position of the forearm when operating a computer mouse reduce the pain level for VDU operators? International Journal of Industrial Ergonomics 30, 307-324.

Agarabi, M., Bonato, P., De Luca, C.J., 2004. A sEMG-based method for assessing the design of computer mice. In: Proceedings of the 26th Annual International Conference of the IEEE Engineering in Medicine and Biology Society, San Francisco, CA, USA, pp. 2450-2453.

Andersen, J.H., Thomsen, J.F., Overgaard, E., Lassen, C.F., Brandt, L.P.A., Vilstrup, I., Kryger, A.I., Mikkelsen, S., 2003. Computer use 
and carpal tunnel syndrome: a 1-year follow-up study. Journal of the American Medical Association 289, 2963-2969.

Aptel, M., Aublet-Cuvelier, A., Cnockaert, J.C., 2002. Work-related musculoskeletal disorders of the upper limb. Joint Bone Spine 69, 546555 .

Atkinson, S., Woods, V., Haslam, R.A., Buckle, P., 2004. Using nonkeyboard input devices: interviews with users in the workplace. International Journal of Industrial Ergonomics 33, 571-579.

Baillif, R.N., Kimmel, D.L., 1945. Structure and Function of the Human Body. J.B. Lippincott Company,, Philadelphia, USA.

Bao, S., Mathiassen, S.E., Winkel, J., 1995. Normalizing upper trapezius EMG amplitude: comparison of different procedures. Journal of Electromyography and Kinesiology 5, 251-257.

Blatter, B.M., Bongers, P.M., 2002. Duration of computer use and mouse use in relation to musculoskeletal disorders of neck or upper limb. International Journal of Industrial Ergonomics 30, 295-306.

Buckle, P.W., 1997. Work factors and upper limb disorders. British Medical Journal 315, 1360-1363.

Burgess-Limerick, R., Shemmell, J., Scadden, R., Plooy, A., 1999. Wrist posture during computer pointing device use. Clinical Biomechanics $14,280-286$.

Clancy, E.A., Morin, E.L., Merletti, R., 2002. Sampling, noise-reduction and amplitude estimation issues in surface electromyography. Journal of Electromyography and Kinesiology 12, 1-16.

Cook, C., Burgess-Limerick, R., Chang, S., 2000. The prevalence of neck and upper extremity musculoskeletal symptoms in computer mouse users. International Journal of Industrial Ergonomics 26, 347-356.

Cram, J.R., Kasman, G.S., Holtz, J., 1998. Introduction to Surface Electromyography. Aspen Publishers, Gaithersburg, Maryland, USA

Fagarasanu, M., Kumar, S., 2003. Carpal tunnel syndrome due to keyboarding and mouse tasks: a review. International Journal of Industrial Ergonomics 31, 119-136.

Fagarasanu, M., Kumar, S., Narayan, Y., 2004. Measurement of angular wrist neutral zone and forearm muscle activity. Clinical Biomechanics 19, 671-677.
Gustafsson, E., Hagberg, M., 2003. Computer mouse use in two different hand positions: exposure, comfort, exertion and productivity. Applied Ergonomics 34, 107-113.

Harvey, R., Peper, E., 1997. Surface electromyography and mouse use position. Ergonomics 40, 781-789.

Jensen, C., Finsen, L., Søgaard, K., Christensen, H., 2002. Musculoskeletal symptoms and duration of computer and mouse use. International Journal of Industrial Ergonomics 30, 265-275.

Johnson, P.W., Lehman, S.L., Rempel, D.M., 1996. Measuring muscle fatigue during computer mouse use. In: Proceedings of the 18th Annual International Conference of the IEEE Engineering in Medicine and Biology Society, Amsterdam, Holland, pp. 1454-1455.

Karlqvist, L., Hagberg, M., Selin, K., 1994. Variation in upper limb posture and movement during word processing with and without mouse use. Ergonomics 37, 1261-1267.

Laursen, B., Jensen, B.R., 2000. Shoulder muscle activity in young and older people during a computer mouse task. Clinical Biomechanics 15, S30-S33.

Oksa, J., Ducharme, M.B., Rintamäki, H., 2002. Combined effect of repetitive work and cold on muscle function and fatigue. Journal of Applied Physiology 92, 354-361.

Ortiz-Hernández, L., Tamez-González, S., Matínez-Alcántara, S., Méndez-Ramírez, I., 2003. Computer use increases the risk of musculoskeletal disorders among newspaper office workers. Archives of Medical Research 34, 331-342.

Visser, B., de Korte, E., van der Kraan, I., Kuijer, P., 2000. The effect of arm and wrist supports on the load of the upper extremity during VDU work. Clinical Biomechanics 15, S34-S38.

Werner, R., Armstrong, T.J., Bir, C., Aylard, M.K., 1997. Intracarpal canal pressures: the role of finger, hand, wrist and forearm position. Clinical Biomechanics 12, 44-51.

Zipp, P., Haider, E., Halpern, N., Rohmert, W., 1983. Keyboard design through physiological strain measurements. Applied Ergonomics 14, $117-122$. 